\title{
Functional and Radiological Evaluations of High-Energy Tibial Plateau Fractures Treated with Double- Buttress Plate Fixation
}

\author{
Z. Yu, L. Zheng, Y. Zhang, J. Li, B. Ma \\ Center of Orthopedic Surgery, Orthopedics Oncology Institute of Chinese PLA, Tangdu Hospital, \\ Fourth Military Medical University, Xi'an, P. R. China
}

\begin{abstract}
Objective: This study was designed to evaluate the functional and radiological outcomes of patients with complex tibial plateau fractures treated with doublebuttress plate fixation.

Methods: Sixty five cases of complex (Schatzker type $\mathrm{V}$ and VI) tibial plateau fractures were treated with double-buttress plate fixation in our centre from September 2001 to September 2006 through two separate plate incisions. Fifty four patients were followed up for a period ranging from 12 to 48 months and evaluated for the functional and radiological outcomes by a series of standard questionnaire and measurement.

Results: Due to the good exposure without any extensive soft-tissue dissection of the double-buttress plate fixation, the fractures in all 54 patients were healed and the treatment achieved greater than $90 \%$ of satisfactory-to-excellent rates of reduction. The mean time of bone union was 15.4 weeks (range, 12-30 weeks), and the mean time of full weight-bearing was 18.7 weeks (range, 14-26 weeks). At the final follow-up visit, no patients showed knee instability; the mean range of motion was $107.6^{\circ}$ (range, $85^{\circ}-130^{\circ}$ ). For all patients, no statistically significant difference in the functional outcomes was observed between their 6-months and final follow-up visits; or in the radiological findings between their immediate postoperative and final follow-up examinations.

Conclusion: Double-buttress plate fixation is a feasible treatment option for bilcondylar and complex tibial plateau fractures. Although technically demanding, it offers reliable stability without additional postoperative adjuvant external fixation, and at the same time avoids extensive soft tissue dissection, allowing the early painless range of motion.

Key words: Functional Outcome; Radiological Outcome; Tibial Plateau Fracture; Fracture Fixation; Double Buttress Plate

Abbreviations: Open reduction internal fixation (ORIF); Anterior cruciate ligament (ACL); Lateral collateral ligament (LCL); Medial collateral ligament (MCL); Tibial plateau angle (TPA); Femorotibial angle (FTA); Posterior slop angle (PA); Rang of motion (ROM); Computerised tomography (CT); Magnetic resonance imaging (MRI)
\end{abstract}

\section{INTRODUCTION}

High-energy tibial plateau fractures remain a challenge to orthopaedic surgeons, with the bicondylar type (Schatzker type V) and the comminuted type (Schatzker type VI) fractures being the most difficult to treat [1]. The severity of the tibial plateau fracture depends on the energy applied to the limb. The higher the energy to the limb, the more severe and complex the fracture is. Compared to their low-energy counterparts, high-energy fractures have unique patterns of fractures. The bicondylar type fracture involves both the lateral and medial tibial plateaus, usually with lateral depression, meniscal detachment, and anterior cruciate ligament (ACL) avulsions [2]. The comminuted type fracture is characterized by the dissociation of the diaphysis from the metaphysis, often associated with severe soft tissue injuries around the knee that require special clinical consideration [3].

Treatment goals of high-energy tibial plateau fractures are to restore the joint stability, congruity, and alignment with minimal soft tissue dissection to allow early joint motion and weight bearing. However, these aims may be difficult to achieve because of the high complication rates and the poor outcomes associated with the surgical treatment. For example, external fixators often cause permanent joint stiffness due to the restriction of early range of motion following the operation. Traditional open reduction and internal fixation with insertion of a single or double buttress plates through a single incision usually requires extensive stripping of the tenuous soft tissue envelope of the proximal tibia, leading to considerable devascularization of fracture fragments, thus delaying fracture healing and increasing the risks of infection and non-union [4].

In an attempt to improve the treatment of high-energy tibial plateau fractures, improved internal fixation with double buttress plates applied through a medial and a lateral incisions has been widely adopted. This technique allows anatomic joint reduction with minimal iatrogenic soft tissue damage and its associated complications, provides adequate fixation of the fracture, and permits early functional range of motion.

In this study, we evaluated the postoperative functional and radiological outcomes of 54 patients with 
high-energy tibial plateau fractures (Schatzker type $\mathrm{V}$ or type VI) treated with the double-buttress plate fixation through a medial and a lateral incisions.

\section{MATERIALS AND METHODS}

\section{Patients}

From September 2001 to September 2006, 65 patients with complex (Schatzker type V or type VI) tibial plateau fractures were treated with the double-buttress plate fixation in the Orthopedic Surgery Center of the Fourth Military Medical University associated Tangdu Hospital,. The general patient information including the pre-injury status was recorded at admission. All fractures were fixed with a bilcondylar double-plating technique by experienced orthopaedic surgeons. Fifty four of the sixty five patients were investigated for the functional and radiological outcomes, and the other eleven patients were excluded for the following reasons: four patients changed their address and could not be contacted, five patients had open fractures, and the other two patients were polytrauma. The patient demographics and fracture types of the $54 \mathrm{pa}-$ tients were collected preoperatively and are shown in Table 1.

\section{RADiologic StUdies}

The compartment syndrome and the neurovascular status were evaluated by a series of radiographic studies. The degree of maximal joint depression was measured in millimeters before the operation, and the displacement and joint congruity were evaluated with radiographs of anteroposterior, lateral and $45^{\circ}$ oblique views. Computerized tomography (CT) was performed for $75 \%$ of cases as needed. Ligament and meniscal injuries were determined using Magnetic resonance imaging (MRI). The tibial plateau angle (TPA), the femorotibial angle (FTA) and the medial posterior slop angle (PA) were measured on a digital X-ray image marked with a scale [5].

\section{Operative Planning and Surgical Technique}

The soft-tissue envelope was recovered before the extensive incisions were made. The skin should be soft, blisters should be epithelialized, and skin wrinkles should be present. A high thigh tourniquet was routinely used during the operation. A laterally based plate $(4.5 \mathrm{~mm}$, T-shaped or L-shaped) was inserted via a lateral incision. Then a second medial incision was made approximately $1 \mathrm{~cm}$ apart from the posterior border of the tibia. Another plate was inserted through an interval created between the medial head of the gastrocnemius and the pes anserinus tendons, and was positioned at the lower portion of the medial condyle's superior border serving as a buttress plate. During the reduction and fixation, the knee was then put in extension by applying traction. The depressed articular surface was elevated and the resulted bony defect was filled with bone substitute before the fixation. Autograft was routinely used when the bone graft was necessary. The meniscus was repositioned and repaired using outside-in technique or experienced meniscectomy if it was trapped in the joint. Postoperatively continuous passive motion was applied and the active knee flexion begun at $48 \mathrm{~h}$ following the surgery. A functional position brace was used for 4-6 weeks if the fractures were severe comminuted, and a hinged brace was used for 12-16 weeks if the anterior cruciate ligament (ACL) was injured.

\section{FOLLOW-UP}

The fifty four patients were followed up for a period ranging from 12 to 48 months (mean, 23.7 months). The length of surgery and the duration of hospital stay were recorded for each case. At the 6-month postoperative follow-up and the subsequent follow-up visits, patients were asked to attend an outpatient clinic where a survey of the HSS score, the Lysholm score and the Knee Society Clinical Rating Score was registered to assess patients' satisfaction, with a maximum of 100 points for each category. Postoperative A-P Xrays of the knee were taken at week 6 , week 12 , and every 6 to 8 weeks subsequently until bony union occurred, and then every 6 months until 2 years after the operation, or until the removal of the plates. The reduction of the fracture was graded as excellent if the residual depression was $2 \mathrm{~mm}$ or less, satisfactory if the residual depression was between 2 and $5 \mathrm{~mm}$, and poor if the depression was greater than $5 \mathrm{~mm}$. Malreduction was defined as intraarticular step-off of 5 $\mathrm{mm}$ or more, or a $\mathrm{TPA} \geq 95^{\circ} / \mathrm{TPA} \leq 80^{\circ}$, or $\mathrm{PA} \geq$ $15^{\circ} / \mathrm{PA} \leq-5^{\circ}$. Radiological bony union was defined by the corresponding surgeon as at least three cortical unions. The type and number of implants used were noted in each case. Complications requiring further surgery were also recorded.

\section{STATISTICAL ANALYSIS}

SPSS12.0 was used for data variation analysis. The mean and the standard deviation for baseline descriptive characteristics were calculated. Statistical comparisons between 6-month postoperative and final followup HSS, Lysholm, and Knee Society Clinical Rating scores were made using paired Student's t-test, with statistical significance set at $\mathrm{P}<0.05$. Similarly, statistical comparisons between the 6-month postoperative and the final follow-up TPA, PA, FTA were also conducted using paired Student's t-test, and $\mathrm{P}<0.05$ was considered statistically significant. The satisfactory-toexcellent rate of reduction was compared using chisquare test $\left(\chi^{2}\right.$ test).

\section{RESULTS}

The surgical details are shown in Table 2.

\section{Clinical Follow-up Evaluations}

A follow up ranging from 12 to 48 months (mean, 23.7 months) showed that all fractures were healed. The mean time between the operation and bone union was 15.4 weeks (range, 12-30 weeks), and the mean interval between the operation and full weight-bearing was 18 
Table 1. Patient demographics and fracture types.

\begin{tabular}{ll}
\hline Details & Number (\%) \\
\hline Age (years) & 45.2 (rang 19-68) \\
Side & Left: $29(53.7)$; right $25(46.3)$ \\
Gender & Female $21(38.9)$, male $33(61.1)$ \\
Isolated fracture & $42(77.8)$ \\
Multiple fractures & $12(22.2)$ \\
Fracture pattern (Schatzker classification) & Schatzker V 23 (42.6), Schatzker VI 31 (57.4) \\
\hline
\end{tabular}

Table 2. Surgical details of the 54 cases of high-energy tibial plateau fractures.

\begin{tabular}{ll}
\hline Details & Number (\%) \\
\hline Average time to surgery & 10.4 days (rang: 1-19 days) \\
Preoperative preparation & Traction: 19 (35.2); bridging external fixator or brace: 30 (55.6); emergency \\
& operation: 5 (9.3) \\
Mean operation time (minutes) & $158.4(100-270)$ \\
Bone graft & Autograft: 37 (64.8); Allograft: 0 \\
Meniscus repair or excised & Yes: 19 (35.2); no: 35 (64.8) \\
ACL inertion fixation & Yes: 6 (11.1); no: 48 (88.9) \\
Lateral implant & $4.5-T-$-shaped: 20 (37.0); 4.5-L-shaped: 13 (24.1); 3.5-LCDCP: 21 (38.9) \\
Medial implant & $3.5-L C D C P: 12$ (22.2); 4.5-T-shaped: 25 (46.3); 4.5-L-shaped: 17 (31.5) \\
Postoperative management & Early exercise 34 (63.0); Traction: 7 (12.9); external brace: 13 (24.1) \\
\hline
\end{tabular}

Table 3. Clinical summary of the 54 cases of high-energy tibial plateau fractures.

\begin{tabular}{ll}
\hline Details & Number \\
\hline Mean duration of follow-up (months) & 23.7 (rang: 12-48) \\
Mean ROM & $107.6^{\circ}\left(85^{\circ}-130^{\circ}\right)$ \\
Mean time of bone union (weeks) & 15.4 (range 12-30) \\
Mean time of full weight-bearing (weeks) & 18.7 (range 14-35)
\end{tabular}

Table 4. Functional scores of the patients .

\begin{tabular}{llll}
\hline & Postoperative $(6$ months $)$ & Final follow-up & P value \\
\hline HSS score & $81.0($ range $42-94)$ & $77.3($ range $43-92)$ & $>0.05$ \\
Lysholm score & $83.2($ range $55-96)$ & $79.5($ range 56-95) \\
Knee Society Clinical Rating Score & 76.2 (range 39-90) & $70.3($ range 39-91) \\
\hline
\end{tabular}

weeks (range, 14-26 weeks). At the final clinical followup, no patients showed knee instability, and the mean range of motion was $107.6^{\circ}\left(85^{\circ}-130^{\circ}\right)$ (Table 3). There were 9 cases suffering from stiffness of knee joint, 3 cases of varus abnormity, 2 cases of valgus abnormity, 2 cases of incisional wound infection (debridement and external fixation were performed with internal implants removed), 10 cases of traumatic arthritis of knee joint ( 2 cases received the total knee arthroplasty 1 year and 2 years postoperatively, and the other 8 cases did not receive any further operation during the follow-up periods), and 1 case of delayed union.

\section{FUNCTIONAL EVALUATIONS}

At the 6-month follow-up, the mean HSS score, the mean Lysholm score, and the mean Knee Society Clinical Rating Score were 81.0 (range, 42-94), 83.2 (range, 55-96), and 76.2 (range, 39-90), respectively. At the final follow-up, these three scores were 77.3 (range, 43-92), 79.5 (range, 56-95), and 70.3 (range, 39-91), respectively. Statistic analysis showed that there was no significant difference between the 6-month postoperative score and the final follow-up score in all the three score systems (Table 4). 
Table 5. Radiological outcomes of the patiens.

\begin{tabular}{|c|c|c|c|}
\hline & Postoperative & Final follow-up & P value \\
\hline The satisfactory-to-excellent rate & $94.4 \%(51 / 54)$ & $90.7 \%(49 / 54)$ & $>0.05$ \\
\hline Mean TPA & $86.0^{\circ} \pm 4.2^{\circ}$ & $85.3^{\circ} \pm 4.0^{\circ}$ & $>0.05$ \\
\hline Mean PA & $9.4^{\circ} \pm 3.3^{\circ}$ & $9.3^{\circ} \pm 3.8^{\circ}$ & $>0.05$ \\
\hline Mean FTA & $176^{\circ} \pm 2.0^{\circ}$ & $175^{\circ} \pm 2.6^{\circ}$ & $>0.05$ \\
\hline
\end{tabular}

TPA: tibial plateau angle; PA: posterior slope angle; FTA: femorotibial angle.

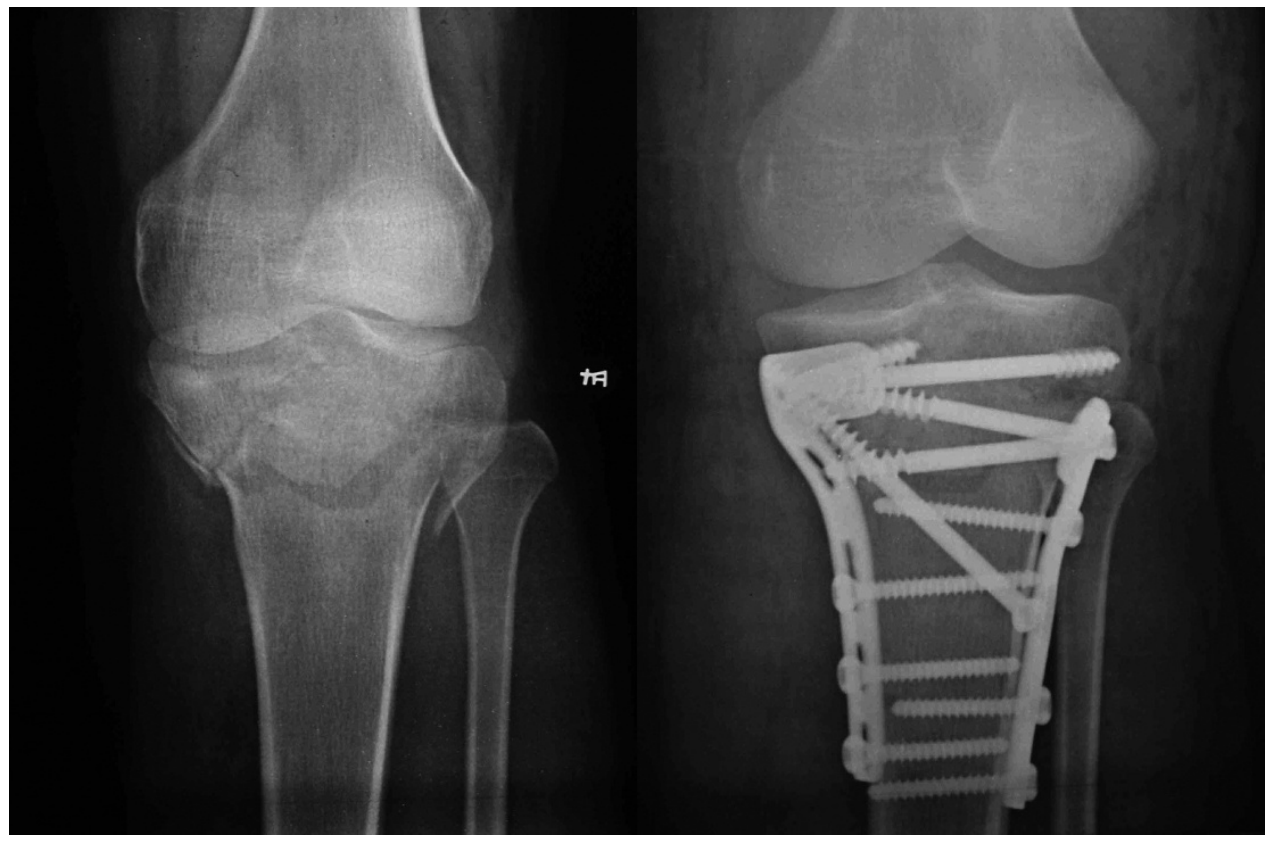

Fig. 1. Left: a radiograph showing the bicondylar tibial plateau fracture (Schatzker Type V). Right: a radiograph taken one year after the operation showing the anatomic reduction.

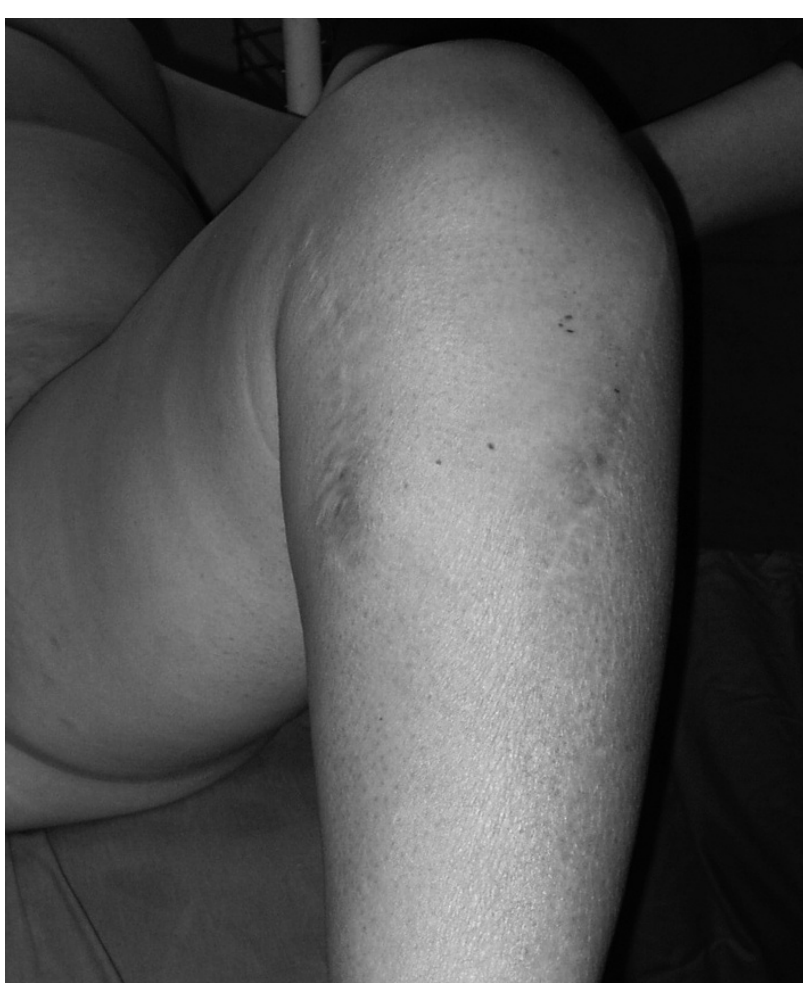

Fig. 2. A patient with the tibial plateau fracture (Schatzker Type V) before surgical removal of the double buttress plates.

\section{RADiOLOGICAL EVALUATIONS}

Immediate postoperative imaging showed the satisfactory-to-excellent rate of reduction was $94.4 \%$, the mean TPA was $86.0^{\circ} \pm 4.2^{\circ}$, PA was $9.4^{\circ} \pm 3.3^{\circ}$, and FTA was $176^{\circ} \pm 2.0^{\circ}$. Subsequent postoperative A-P $\mathrm{X}$-rays of the knee were taken at week 6 , week 12 , and every 6 to 8 weeks until bony union occurred, and then taken every 6 months until 2 years after the operation or until the removal of the plates. A-P X-rays taken at the final follow-up showed the satisfactory-to-excellent rate of reduction was $90.7 \%$, the mean TPA was $85.3^{\circ}$ $\pm 4.0^{\circ}$, PA was $9.3^{\circ} \pm 3.8^{\circ}$ and FTA was $175^{\circ} \pm 2.6^{\circ}$. Statistically, no significant difference was observed between the immediate postoperative imaging and the final follow-up imaging (Table 5) (Fig. 1, 2).

\section{DisCUSSION}

The tibial plateau fracture involves the major weightbearing knee joint. To preserve normal knee function, treatment must aim to reestablish joint stability, alignment, and articular congruity to ensure a full range of motion. The treatment of tibial plateau fractures can be nonoperative or operative. Nonoperative treatment is a simple technique using traction mobilization or functional cast bracing but it has the potential risk of complications associated with prolonged traction or other forms of immobilization such as decubitus ul- 
cers, urinary tract infections, pneumonias, muscle atrophy, contractures, mental deterioration, leading to a prolonged hospital stay. The advantages of the operative treatment such as anatomical reduction and early mobilization have been emphasized by many recent reports $[6,7]$. Operative treatment includes internal and external fixation, hybrid fixation and arthroscopically assisted techniques. Among them, double-buttress plating represented open reduction and internal fixation technique gains the greatest popularity because of its advantages in diminishing the risks of articular incongruence, malalignment of mechanical load axis, and post-traumatic degenerative joint disease [8].

Although external fixation techniques using unilateral fixator, single-ring fixator, two-ring fixator, and circular frames (Ilizarov fixator) have been shown to treat high-energy tibial plateau fractures with severe soft tissue injuries effectively [9, 10],none of the above techniques except the two-ring fixation are comparable to double-buttress plating technique in terms of stability [11]. Some authors believe that open reduction and internal fixation with plates and screws will bring a high rate of complications including deep infection, wound dehiscence and soft tissue problems [12]. However, in complex tibial plateau fractures, it is mandatory to anatomically reduce the articular part of the fracture in order to prevent the secondary arthritis and achieve a stable articular and metaphyseal fixation to facilitate the fracture healing regardless which treatment option is selected [13]. Moreover, soft tissue complications can be largely minimized by staging the treatment of the patient.

Bilcondylar tibial plateau fracture dislocation is a very unstable and serious condition caused by high-energy injury, and successful treatment requires an understanding of the fracture patterns. A single anterolateral or posteromedial approach makes both reduction and fixation of this unstable fracture pattern difficult [14]. Although a midline anterior approach using the same incision as that in the traditional total knee arthroplasty permits simultaneous exposure of both plateaus to facilitate later salvage arthroplasty, it unavoidably involves extensive soft-tissue dissection that will compromise the viability of the overlying soft tissue and devitalizes the underlying bone, resulting in an unacceptable incidence of soft tissue necrosis, wound dehiscence and deep infection [15]. With open reduction and internal fixation technique, the main fracture parts and the medial plateau are easily accessed through a lateral and a medial incisions with minimal soft tissue dissection.

Locking plates have gained popularity in recently years because of the improved rigidness. A single locking plate is inserted submuscularly through a limited incision with percutaneously placed locking screws to minimize soft tissue injury. However, Schatzker type V and VI tibial plateau fractures are the most comminuted and complicated types that involve both the joint surface damage and cancellous bone compression. If the incision is too small, fluoroscopic or direct visual evaluation of the anatomical reduction cannot be achieved [16]. Additionally, the position of the locking plate cannot be adjusted freely to fit the fracture pattern perfectly since the direction of the screw is pre- determined by the thread in the plate. Studies have shown that a lateral locking plate is not always sufficient to protect the fracture from collapse, and an additional medial plate is necessary to further stabilize the fracture [17].

Another benefit of the double-buttress plating technique is that it makes the adjuvant external fixation (brace or plaster) unnecessary. As for severe comminuted tibial plateau fractures, traditional single-buttress plate fixation usually needs adjuvant external fixation to ensure the rigid immobile condition [18, 19]. However, prolonged cast immobilization of the knee often leads to some degree of permanent stiffness, thus it has been abandoned in our routine practice in the centre. The best treatment of patients who fail to regain knee motion during the first month following the surgery is passive continuous motion or other aggressive range-of-motion exercises under the instruction of a physical therapist. In our series, no iatrogenic displacements were found in any of the patients.

Soft tissue management is also a very important issue in the treatment of high-energy tibia plateau fractures as injuries often involved soft tissues, especially ACL, lateral collateral ligament (LCL), medial collateral ligament (MCL) and meniscus. Six patients suffered from ACL insertion avulsion fracture and were treated with ACL insertion fixation with screws. As the avulsion insertions were all fresh fractures, ACL reconstruction using patellar ligament, or gracilis and semitendinosus tendons was not required. After the ACL insertion fixation, a hinged brace was used for 12-16 weeks to protect the fixed ACL. LCL and MCL injuries are also common in the patients, but neither of them is complete, nor need any surgery.

\section{CONCLUSION}

This study, for the first time in China, evaluated the treatment efficacy of double-buttress plate fixation technique in high-energy Schatzker V and VI tibial plateau fractures. The postoperative functional and radiological outcomes indicate that this technique is a feasible treatment option for bicondylar and complex tibial plateau fractures. Our results are consistent with the international standards. Although technically demanding, the double-buttress plate fixation provides reliable stability without additional postoperative adjuvant external fixation. The potential postoperative complications associated with internal fixation can be reduced by practices including careful timing of surgery, extraperiosteal dissection and limited dissection of comminuted bone fragments.

Acknowledgements: This study was supported by the National Natural Science Foundation (30330610, CHN). We thank Dr. Yunfei Zhang for the assistance with the data processing.

\section{REFERENCES}

1. Ali AM, Yang L, Hashmi M, Saleh M. Bicondylar tibial plateau fractures managed with the Sheffield Hybrid Fixator. Biomechanical study and operative technique. Injury. 2001; 32: SD86-91.

2. Berkson EM, Virkus WW. High-energy tibial plateau fractures. J Am Acad Orthop Surg. 2006; 14:20-31. 
3. Papagelopoulos PJ, Partsinevelos AA, Themistocleous GS, Mavrogenis AF, Korres DS, Soucacos PN. Complications after tibia plateau fracture surgery. Injury. 2006; 37: 475-484.

4. Chin TY, Bardana D, Bailey M, Williamson OD, Miller R, Edwards ER, Esser MP. Functional outcome of tibial plateau fractures treated with the fine-wire fixator. Injury. 2005; 36: 1467-1475.

5. Luo CF, Jiang R, Hu CF, Zeng BF. Medial double-plating for fracture dislocations involving the proximal tibia. Knee. 2006; 13: 389-394.

6. Barei DP, Nork SE, Mills WJ, Coles CP, Henley MB, Benirschke SK. Functional outcomes of severe bicondylar tibial plateau fractures treated with dual incisions and medial and lateral plates. J Bone Joint Surg Am. 2006; 88: 1713-1721.

7. Wu CC. Salvage of proximal tibial malunion or nonunion with the use of angled blade plate. Arch Orthop Trauma Surg. 2006; 126: 82-87.

8. Chin TY, Bardana D, Bailey M, Williamson OD, Miller R, Edwards ER, Esser MP. Functional outcome of tibial plateau fractures treated with the fine-wire fixator. Injury. 2005; 36:1467-1475.

9. Piper KJ, Won HY, Ellis AM. Hybrid external fixation in complex tibial plateau and plafond fractures: an Australian audit of outcomes. Injury. 2005; 36: 178-184.

10. Catagni MA, Ottaviani G, Maggioni M. Treatment strategies for complex fractures of the tibial plateau with external circular fixation and limited internal fixation. J Trauma. 2007; 63: 1043-1053.

11. Ali AM, Saleh M, Bolongaro S, Yang L. The strength of different fixation techniques for bicondylar tibial plateau fractures--a biomechanical study. Clin Biomech (Bristol, Avon). 2003;18: 864-870.

12. Stannard JP, Robinson JT, Anderson ER, McGwin G Jr, Volgas DA, Alonso JE. Negative pressure wound therapy to treat hematomas and surgical incisions following highenergy trauma. J Trauma. 2006; 60:1301-1306.

13. Kiene J, Schulz AP, Hillbricht S, Jürgens Ch, Paech A. Clinical results of resection arthrodesis by triangular external fixation for posttraumatic arthrosis of the ankle joint in 89 cases. Eur J Med Res. 2009; 14: 25-29.
14. Higgins TF, Kemper D, Klatt J. Incidence and morphology of the posteromedial fragment in bicondylar tibial plateau fractures. J Orthop Trauma. 2009; 23: 45-51.

15. Shah SN, Karunakar MA. Early wound complications after operative treatment of high energy tibial plateau fractures through two incisions. Bull NYU Hosp Jt Dis. 2007; 65: 115-119.

16. Weil YA, Gardner MJ, Boraiah S, Helfet DL, Lorich DG. Posteromedial supine approach for reduction and fixation of medial and bicondylar tibial plateau fractures. J Orthop Trauma. 2008; 22: 357-362.

17. Higgins TF, Klatt J, Bachus KN. Biomechanical analysis of bicondylar tibial plateau fixation: how does lateral locking plate fixation compare to dual plate fixation? J Orthop Trauma. 2007; 21:301-306.

18. Marti RK, Kerkhoffs GM, Rademakers MV. Correction of lateral tibial plateau depression and valgus malunion of the proximal tibia. Oper Orthop Traumatol. 2007; 19: 101-113.

19. Mann ST, Mann V, Stracke H, Lange U, Klör HU, Hardt $\mathrm{P}$, Teichmann J. Fecal elastase 1 and vitamin D3 in patients with osteoporotic bone fractures. Eur J Med Res. 2008; 13: 68-72.

Received: March 22, 2009 / Accepted: March 26, 2009

Address for correspondence:

Bao'an Ma

Center of Orthopedic Surgery

Orthopedics Oncology Institute of Chinese PLA

Tangdu Hospital

Fourth Military Medical University

Xi'an

Shanxi, 710038

P. R. China

Tel.: $\quad+8629 / 84777433$

Fax: $\quad+8629 / 83549796$

E-mail: yuzhe780519@yahoo.com.cn 